\title{
Indicações da neurotomia dos nervos geniculares por radiofrequência para o tratamento da osteoartrite do joelho: uma revisão de literatura*
}

\section{Indications of the Neurotomy of Genicular Nerves by Radiofrequency for the Treatment of Knee Osteoarthritis: A Literature Review}

\author{
Diego Ariel de Lima ${ }^{1,2}$ Marcelo Carvalho Krause Gonçalves ${ }^{1}$ Souterland Thiago Correia e Sá Grando ${ }^{1}$ \\ Thiago Luiz de Lima Cintra ${ }^{1}$ Dilamar Moreira Pinto ${ }^{1}$ Romeu Krause Gonçalves ${ }^{1}$ \\ ${ }^{1}$ Grupo de Joelho do Instituto de Traumatologia e Ortopedia Romeu \\ Krause (ITORK), Recife, PE, Brasil \\ 2 Universidade Federal Rural do Semi-Árido (UFERSA), Mossoró, RN, Brasil \\ Address for correspondence Diego Ariel de Lima, MD, PhD, \\ Universidade Federal Rural do Semi-Árido (UFERSA), Av João da \\ Escóssia, 1300, casa 124, Mossoró, RN 59607-330, Brasil \\ (e-mail: arieldelima.diego@gmail.com).
}

Rev Bras Ortop 2019;54:233-240.

\section{Resumo \\ Palavras-chave \\ - procedimentos neurocirúrgicos \\ - ondas de rádio \\ - rizotomia \\ - osteoartrite do joelho \\ - articulação do joelho}

A osteoartrite é uma das patologias mais frequentes e incapacitantes na atualidade, principalmente do joelho. Dentre as abordagens possíveis para osteoartrite, a neurotomia dos nervos geniculares por radiofrequência vem se destacando. Todavia, por se tratar de um procedimento relativamente novo, as indicações para realização ainda não estão bem definidas. O principal objetivo desta revisão foi identificar as principais indicações do uso da radiofrequência para o tratamento da osteoartrite do joelho na literatura médica. Foi realizada revisão da literatura em janeiro de 2018, através de pesquisa nas bases de dados PubMed, Clinicalkey e Google Scholar. Após revisão dos principais artigos no assunto, foi concluído que as principais indicações do uso da radiofrequência para o tratamento da osteoartrite do joelho foram: pacientes com osteoartrite grau 3 e 4 da classificação de Kellgren-Lawrence, com dor de moderada a severa e falha do tratamento conservador, principalmente idosos; persistência da dor, mesmo após realizado artroplastia total de joelho; pacientes com indicação de artroplastia total de joelho e que se recusam a submeter-se ao tratamento cirúrgico.

\section{Abstract \\ Osteoarthritis is one of the most frequent and incapacitating pathologies today, especially of the knee. Among the possible approaches for knee osteoarthritis, the neurotomy of the genicular nerves by radiofrequency has been gaining prominence. However, as this is a relatively new procedure, indications for its implementation are}

Trabalho realizado no Instituto de Traumatologia e Ortopedia Romeu Krause, Recife, PE, Brasil.

(1)Diego Ariel de Lima's ORCID is https://orcid.org/0000-00025702-1529.

received

January 15, 2018

accepted

May 7, 2018
DOI https://doi.org/

10.1055/s-0039-1692121. ISSN 0102-3616.
Copyright $\odot 2019$ by Sociedade Brasileira License terms de Ortopedia e Traumatologia. Published by Thieme Revnter Publicações Ltda, Rio de Janeiro, Brazil 
Keywords

- neurosurgical procedures

- radio waves

- rhizotomy

- osteoarthritis, knee

- knee joint still unclear. The objective of this review is to identify the main indications of the use of radiofrequency for the treatment of knee osteoarthritis in the medical literature. A review of the literature was performed in January 2018, through a search in PubMed, Clinicalkey and Google Scholar databases. After reviewing the main articles in the subject, it was concluded that the main indications of the use of radiofrequency for the treatment of knee osteoarthritis were: osteoarthritis grade 3 and 4 KellgrenLawrence, with moderate to severe pain and failure of conservative treatment, mainly elderly people; Persistence of pain even after total knee arthroplasty; Patients with an indication for total knee arthroplasty who refuse to undergo surgical treatment.

\section{Introdução}

A osteoartrite $(\mathrm{OA})$ é uma patologia de origem multifatorial que acarreta degeneração da cartilagem articular, afetando os componentes da articulação acometida. Constitui distúrbio musculoesquelético, geralmente insidioso, progressivo e lento, que atinge tipicamente as articulações das mãos, coluna, quadril e joelho, prejudicando a capacidade laboral e as atividades da vida diária desses pacientes. É o distúrbio articular mais comum, podendo acometer de $6 \%$ a $12 \%$ da população adulta e mais de um terço das pessoas com mais de 65 anos de idade. ${ }^{1,2}$

A progressão da OA de joelho é o motivo mais comum para substituição total desta articulação. Além disso, é um dos mais importantes fatores de gasto com saúde em nossa sociedade. ${ }^{3}$

Como o principal objetivo de tratamento da osteoartrite de joelho é proporcionar alívio da dor e melhorar o estado funcional dos pacientes, é necessária abordagem multidisciplinar, em vista de melhores resultados funcionais. ${ }^{4-6}$

A abordagem inicial da OA é procedida com o tratamento não cirúrgico, ou seja, conservador. É realizado através do uso de medicação analgésica e mudanças de estilo de vida, como redução de peso, exercício físico, fisioterapia e até acupuntura. ${ }^{3}$ Os anti-inflamatórios, geralmente, são reservados para o resgate nas crises agudas. Outras medicações são frequentemente utilizadas, destacando-se a glicosamina, a condroitina, extrato insaponificável de soja e de abacate, a diacereína, o colágeno e a viscossuplementação com ácido hialurônico que, muitas vezes, apresenta resultados conflitantes e inconsistentes na literatura. ${ }^{1,7-10}$

O tratamento cirúrgico está indicado na falha do tratamento conservador e deve acontecer nos casos de acometimento progressivo da independência das atividades de vida diária. As cirurgias disponíveis são: desbridamento artroscópico, osteotomias, artroplastias e artrodeses. ${ }^{11,12}$

Atualmente, o tratamento ablativo com radiofrequência (RF), na temperatura de 80 a $90^{\circ} \mathrm{C}$, tem sido utilizado para o tratamento de várias condições dolorosas, como nevralgia do trigêmeo, tratamento sintomático de dores de causa oncológica e dor facetaria na coluna vertebral. ${ }^{13-16}$

A RF trata-se de uma corrente elétrica alternada com frequência oscilatória de $500.000 \mathrm{hz}$, a qual gera o calor necessário para lesão neuronal desejada. Além da RF ablativa convencional, pode-se utilizar RF pulsada (temperatura até $45^{\circ} \mathrm{C}$ ) e a RF ablativa refrigerada. ${ }^{16,17}$
No joelho, o principal alvo da RF consiste na inervação sensorial peri ou intra-articular, através dos ramos geniculares.

Nos últimos cinco anos, algumas publicações científicas vêm sugerindo papel importante da RF no tratamento da $\mathrm{OA}$ de joelho e nos casos de dor persistente pós artroplastia total de joelho (ATJ). ${ }^{16}$

Por se tratar de método novo, é fundamental reunir as principais evidências cientificas da atualidade, para que se possa verificar a real importância da RF no tratamento da gonartrose.

Assim, o principal objetivo desta revisão foi identificar as principais indicações do uso da RF para o tratamento da OA do joelho na literatura médica.

\section{Metodologia}

Pesquisa eletrônica foi realizada em janeiro de 2018 por dois dos autores (MCKG e, DAL), nas bases de dados do PubMed, Clinicalkey e Google Scholar, com limite de data nos últimos cinco anos.

Os seguintes termos de indexação foram usados para a busca: "knee radiofrequency neurotomy," "knee rhizotomy," "knee radiofrequency Ablation," "genicular neurotomy" e "knee neurolysis."

Foram utilizados os títulos e resumos para selecionar artigos que responderam ao objetivo de pesquisa. Assim, apenas os artigos que apresentassem estudos com indicação de realização de rizotomia genicular nos títulos ou resumos foram selecionados. Os artigos selecionados foram lidos em versão completa e suas listas de referência foram pesquisadas manualmente em busca de adicionais publicações relevantes. As discrepâncias na extração de dados foram resolvidas por meio de discussão entre os autores.

Como critério de inclusão, apenas estudos clínicos que apresentassem indicações de realização de rizotomia genicular foram selecionados. Somente os artigos que possuíam versão completa ou pelo menos o resumo em inglês foram incluídos. Foram excluídos os estudos nos quais os pacientes foram seguidos por menos de três meses e os artigos de estudos puramente anatômicos e relato de caso, assim como artigos de revisão que não continham dados originais.

Os seguintes dados foram pesquisados nos estudos incluídos: indicação de rizotomia, autores e data de publicação, tamanho da amostra, média de idade e seguimento. 


\section{Resultados}

\section{Artigos Encontrados}

Foram encontrados 505 artigos no PubMed [knee radiofrequency neurotomy (437), knee rhizotomy (08), knee radiofrequency Ablation (41), genicular neurotomy (5) e knee neurolysis (14)], 521 no ClinicalKey [knee radiofrequency neurotomy (67), knee rhizotomy (23), knee radiofrequency Ablation (281), genicular neurotomy (11) e knee neurolysis (139)] e 4341 no Google Scholar [knee radiofrequency neurotomy (990), knee rhizotomy (198), knee radiofrequency Ablation (952), genicular neurotomy (191) e knee neurolysis (2010)].

Após excluir os artigos que estavam indexados simultaneamente em mais de uma base de dados, chegou-se ao número de 3.820 artigos.

Após leitura dos títulos e resumos, foi obtido número de 57 artigos. Por fim, após leitura completa dos artigos e aplicação dos critérios de exclusão, finalizou-se a busca, com número total de 19 artigos encontrados (-Fig. 1).

\section{Pacientes}

Os 19 estudos incluíram total de 859 pacientes, a maioria do sexo feminino. A média de idade ficou entre 60 e 70 anos. Todos tiveram avaliação clínica e de imagem antes da indicação da RF.

\section{Seguimento}

Os estudos apresentaram seguimento dos pacientes de pelo menos três meses após o procedimento. Para o acompanhamento clínico, foram utilizadas escalas de mensuração de dor e de funcionalidade, como Visual Analogue Scales (VAS), ${ }^{18}$ The Western Ontario and McMaster Universities Osteoarthritis Index (WOMAC), ${ }^{19}$ Patient's Global Impression (PGI-I), ${ }^{20}$ Knee Society Score (KSS), ${ }^{21}$ Oxford Knee Score (OKS), ${ }^{22}$ Numeric Rating Scale $(\mathrm{NRS})^{23}$ e Goldberg Anxiety and Depression Scale (GADS). ${ }^{24}$

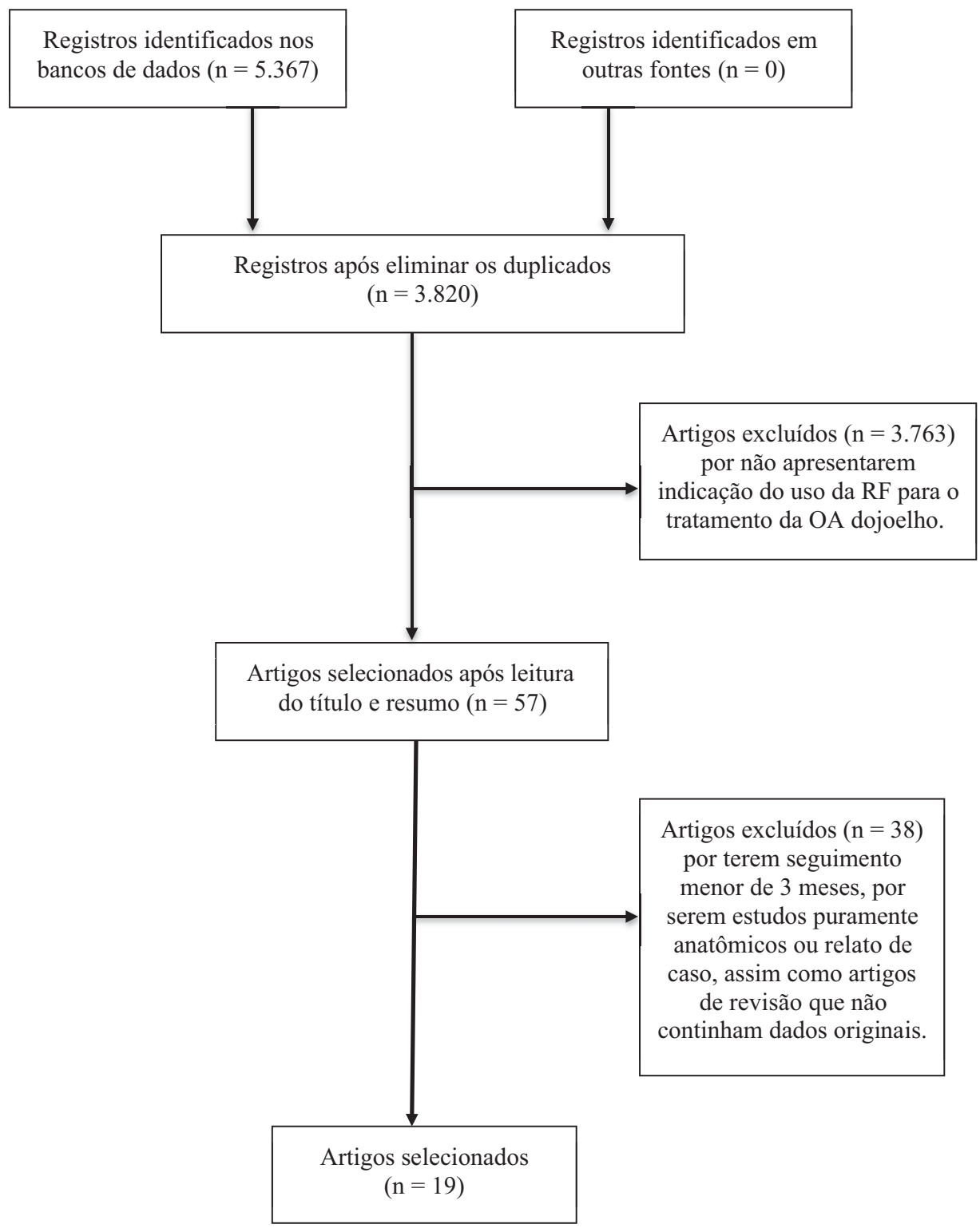

Fig. 1 Fluxograma de seleção dos artigos. Abreviações: OA, osteoartrite; RF, radiofrequência. 


\section{Tipo de RF e Método de Imagem Auxiliar}

Dentre os tipos de RF utilizadas, três modalidades foram utilizadas: ablativa convencional, ablativa refrigerada ou pulsada.

Como método adjuvante para localizar o correto posicionamento dos eletrodos durante a aplicação da RF, foram citados a fluoroscopia e a ultrassonografia.

Indicação de RF

Os estudos evidenciaram as indicações do uso da RF para tratamento da OA do joelho.

As indicações para o uso da RF no tratamento da gonartrose e as respectivas conclusões dos artigos estão listadas na - Tabela $1.25-43$

\section{Resultados do Procedimento}

Entre os trabalhos selecionados, os melhores resultados são obtidos nos seis primeiros meses de acompanhamento. McCormick et al. ${ }^{31}$ relataram completo alívio da dor nesse período.

Após seis meses os resultados vão diminuindo. Iannaccone et al. ${ }^{32}$ referem não mais que $60 \%$ de alívio da dor após seis meses.

Santana Pineda et al. ${ }^{26}$ referem que o efeito do tratamento começou a diminuir após seis meses, mas até um ano após a intervenção, $32 \%$ dos pacientes relataram melhora de $50 \%$ ou mais em resultados de VAS de pré-tratamento. Segundo Bellini et al., ${ }^{29}$ até 12 meses de seguimento revelam alívio clinicamente relevante da dor e melhora funcional.

Tabela 1 Indicação de RF para tratamento da OA de joelho

\begin{tabular}{|c|c|c|}
\hline Autor e Ano & Indicação & Conclusão do Estudo \\
\hline Kirdemir et al, $2017^{25}$ & $\begin{array}{l}\text { Pacientes com osteoartrite grau } 2 \text { a } 4 \text { da } \\
\text { classificação de Kellgren-Lawrence refratários } \\
\text { ao tratamento conservador por } 6 \text { meses. }\end{array}$ & $\begin{array}{l}\text { A neurotomia dos nervos geniculares provo- } \\
\text { cou uma redução significativa da dor e mel- } \\
\text { hora funcional em pacientes idosos com dor } \\
\text { crônica por gonartrose e, portanto, pode ser } \\
\text { um tratamento eficaz em tais casos. }\end{array}$ \\
\hline Pineda et al, $2017^{26}$ & $\begin{array}{l}\text { Pacientes com osteoartrite grau } 3 \text { e } 4 \text { da } \\
\text { classificação de Kellgren-Lawrence com pon- } \\
\text { tuação } \geq 5 \text { na VAS por mais de } 6 \text { meses em } \\
\text { conservador. }\end{array}$ & $\begin{array}{l}\text { Neurotomia dos nervos geniculares por RF } \\
\text { guiada por ultrassom alivia a dor e a incapa- } \\
\text { cidade intratáveis na maioria dos pacientes } \\
\text { com OA avançada do joelho. Tal tratamento é } \\
\text { seguro e minimamente invasivo, podendo ser } \\
\text { realizado em ambiente ambulatorial. }\end{array}$ \\
\hline Sarı et al, $2018^{27}$ & $\begin{array}{l}\text { Pacientes com osteoartrite grau } 2 \text { a } 4 \text { da } \\
\text { classificação de Kellgren-Lawrence refratários } \\
\text { ao tratamento conservador por } 3 \text { meses e } \\
\text { com dor modera e grave não elegíveis para } \\
\text { ATJ. }\end{array}$ & $\begin{array}{l}\text { A neurotomia por RF dos nervos geniculares é } \\
\text { um tratamento seguro e eficiente, propor- } \\
\text { cionando melhorias funcionais, juntamente } \\
\text { com uma analgesia em pacientes com OA } \\
\text { crônica do joelho. }\end{array}$ \\
\hline Kesikburun et al, $2016^{28}$ & $\begin{array}{l}\text { Pacientes com osteoartrite grau } 3 \text { e } 4 \text { da } \\
\text { classificação de Kellgren-Lawrence refratários } \\
\text { ao tratamento conservador por } 6 \text { meses e } \\
\text { que tiveram pelo menos uma redução de } 50 \% \\
\text { na escala da escala VAS após o procedimento } \\
\text { do bloqueio dos nervos geniculares com } \\
\text { solução anestésica. }\end{array}$ & $\begin{array}{l}\text { A neurotomia por RF pulsada dos nervos } \\
\text { geniculares foi considerado segura e benéfica } \\
\text { na dor no joelho associada à OA. }\end{array}$ \\
\hline Bellini et al, $2015^{29}$ & $\begin{array}{l}\text { Pacientes com osteoartrite refratários ao } \\
\text { tratamento conservador por } 3 \text { meses e com } \\
\text { dor modera e grave. }\end{array}$ & $\begin{array}{l}\text { A maioria dos pacientes com dor crônica no } \\
\text { joelho experimentou um alívio clinicamente } \\
\text { relevante da dor e melhora funcional após RF } \\
\text { refrigerada dos nervos geniculares em um, } \\
\text { três, seis e } 12 \text { meses de seguimento. }\end{array}$ \\
\hline Davis et al, $2018^{30}$ & $\begin{array}{l}\text { Pacientes com osteoartrite grau } 2 \text { a } 4 \text { da } \\
\text { classificação de Kellgren-Lawrence refratários } \\
\text { ao tratamento conservador por } 6 \text { meses, com } \\
\text { NRS } \geq 6 \text {, OKS } \leq 35 \text {, uso de opioides ou } \\
\text { equivalentes e que tiveram pelo menos uma } \\
\text { redução de } 50 \% \text { na escala da escala NRS após } \\
\text { o procedimento do bloqueio dos nervos } \\
\text { geniculares com solução anestésica e } \\
\text { corticoide. }\end{array}$ & $\begin{array}{l}\text { A neurotomia por RF refrigerada dos nervos } \\
\text { geniculares é opção terapêutica a longo } \\
\text { prazo para gerenciar dor e melhora da função } \\
\text { e qualidade de vida de pacientes com } \\
\text { gonartrose quando comparado com injeção } \\
\text { de corticoide. }\end{array}$ \\
\hline McCormick et al, $2017^{31}$ & $\begin{array}{l}\text { Pacientes com osteoartrite refratários ao } \\
\text { tratamento conservador e que tiveram uma } \\
\text { melhora após o procedimento do bloqueio } \\
\text { dos nervos geniculares com solução } \\
\text { anestésica. }\end{array}$ & $\begin{array}{l}\text { A neurotomia por RF refrigerada dos nervos } \\
\text { geniculares demonstrou um sucesso de } 35 \% \text { e } \\
\text { 19\% dos procedimentos resultaram em } \\
\text { completa alívio da dor após seis meses de } \\
\text { acompanhamento. }\end{array}$ \\
\hline
\end{tabular}


Tabela 1 (Continued)

\begin{tabular}{|c|c|c|}
\hline Autor e Ano & Indicação & Conclusão do Estudo \\
\hline lannaccone et al, $2017^{32}$ & $\begin{array}{l}\text { Pacientes com osteoartrite refratários ao } \\
\text { tratamento conservador e que tiveram uma } \\
\text { melhora de pelo menos } 80 \% \text { após o procedi- } \\
\text { mento do bloqueio dos nervos geniculares } \\
\text { com solução anestésica. }\end{array}$ & $\begin{array}{l}\text { A neurotomia por RF refrigerada dos nervos } \\
\text { geniculares pode fornecer, em média, mais } \\
\text { de } 60 \% \text { de alívio da dor em um seguimento de } \\
6 \text { meses. }\end{array}$ \\
\hline Qudsi-Sinclair et al, $2017^{33}$ & $\begin{array}{l}\text { Pacientes com persistência de dor, por pelo } \\
\text { menos } 6 \text { meses, após ATJ e refratários ao } \\
\text { tratamento conservador. }\end{array}$ & $\begin{array}{l}\text { Mais estudos são necessários para avaliar a } \\
\text { resposta a longo prazo }\end{array}$ \\
\hline Sarı et al, $2017^{34}$ & $\begin{array}{l}\text { Pacientes com osteoartrite grau } 2 \text { a } 4 \text { da } \\
\text { classificação de Kellgren-Lawrence refratários } \\
\text { ao tratamento conservador por } 3 \text { meses. }\end{array}$ & $\begin{array}{l}\text { Os resultados da neurotomia por RF com o } \\
\text { uso de usltrassom e da fluoroscopia para } \\
\text { ajudar no procedimento são semelhantes. }\end{array}$ \\
\hline Shen et al, $2017^{35}$ & $\begin{array}{l}\text { Pacientes com persistência de dor decorrente } \\
\text { de OA por pelo menos } 3 \text { meses e VAS } \geq 6 \text {. }\end{array}$ & $\begin{array}{l}\text { A neurotomia por RF dos nervos geniculares é } \\
\text { mais efetiva que o tratamento regular para } \\
\text { aliviar a dor refratária e promover a recuper- } \\
\text { ação da função em pacientes com OA de } \\
\text { joelho. }\end{array}$ \\
\hline Mogahed et al, $2017^{36}$ & $\begin{array}{l}\text { Pacientes com osteoartrite refratários ao } \\
\text { tratamento conservador por } 3 \text { meses e VAS } \\
>5 \text { não elegíveis para AT]. }\end{array}$ & $\begin{array}{l}\text { Ambas, a neurotomia por RF convencional e } \\
\text { pulsada controlam a dor em pacientes com } \\
\text { OA de joelho, diminuindo a quantidade de } \\
\text { consumo de medicação analgésica. }\end{array}$ \\
\hline Mata et al, $2017^{37}$ & $\begin{array}{l}\text { Pacientes com osteoartrite grau } 2 \text { a } 4 \text { da } \\
\text { classificação de Kellgren-Lawrence refratários } \\
\text { ao tratamento conservador por } 6 \text { meses e } \\
\text { VAS } \geq 4 \text { por mais de } 3 \text { meses. }\end{array}$ & $\begin{array}{l}\text { Ainda não concluiu, mas recomenda que mais } \\
\text { estudos são necessários para avaliar a } \\
\text { resposta a longo prazo }\end{array}$ \\
\hline Gulec et al, $2017^{38}$ & $\begin{array}{l}\text { Pacientes com osteoartrite grau } 2 \text { e } 3 \text { da } \\
\text { classificação de Kellgren-Lawrence com dor } \\
\text { por pelo menos } 3 \text { meses. }\end{array}$ & $\begin{array}{l}\text { A RF bipolar é mais vantajosa na redução da } \\
\text { dor crônica do joelho e recuperação funcional } \\
\text { em comparação com RF unipolar. Mais estu- } \\
\text { dos são necessários. }\end{array}$ \\
\hline Masala et al, $2014^{39}$ & $\begin{array}{l}\text { Pacientes com osteoartrite grau } 3 \text { e } 4 \text { da } \\
\text { classificação de Kellgren-Lawrence refratários } \\
\text { ao tratamento conservador por } 6 \text { meses. }\end{array}$ & $\begin{array}{l}\text { A neurotomia por RF pulsada dos nervos } \\
\text { geniculares parece ser uma técnica efetiva e } \\
\text { confiável para gerenciamento paliativo da dor } \\
\text { crônica em pacientes com OA do joelho. }\end{array}$ \\
\hline Hashemi et al, $2016^{40}$ & $\begin{array}{l}\text { Pacientes com osteoartrite grau } 2 \text { e } 3 \text { da } \\
\text { classificação de Kellgren-Lawrence refratários } \\
\text { ao tratamento conservador por } 3 \text { meses. }\end{array}$ & $\begin{array}{l}\text { A neurotomia por RF dos nervos geniculares e } \\
\text { a ozonioterapia intra-periarticular são boas } \\
\text { indicações clínicas na OA do joelho, com } \\
\text { superioridade da RF em idade acima de } 65 \\
\text { anos. }\end{array}$ \\
\hline Ramírez Ogalla et al, $2014^{41}$ & $\begin{array}{l}\text { Pacientes com osteoartrite grau } 3 \text { e } 4 \text { da } \\
\text { classificação de Kellgren-Lawrence refratários } \\
\text { ao tratamento conservador por } 3 \text { meses e } \\
\text { VAS } \geq 5 \text {. }\end{array}$ & $\begin{array}{l}\text { A neurotomia por RF dos nervos geniculares } \\
\text { mostrou benefício significativo em termos de } \\
\text { redução da dor e melhoria funcional em um } \\
\text { acompanhamento de } 6 \text { meses em pacientes } \\
\text { com OA crônica do joelho e, portanto, pode } \\
\text { ser um tratamento eficaz em tais casos. São } \\
\text { necessários ensaios adicionais com tamanho } \\
\text { amostral maior e acompanhamento mais } \\
\text { longo. }\end{array}$ \\
\hline Eyigor et al, $2015^{42}$ & $\begin{array}{l}\text { Pacientes com osteoartrite grau } 3 \text { da classi- } \\
\text { ficação de Kellgren-Lawrence refratários ao } \\
\text { tratamento conservador. }\end{array}$ & $\begin{array}{l}\text { É possível afirmar que a neurotomia por RF } \\
\text { dos nervos geniculares é eficaz e segura para } \\
\text { o tratamento da dor de pacientes com OA de } \\
\text { joelho avançada. Portanto, pensamos que tal } \\
\text { procedimento será incluído nas diretrizes de } \\
\text { tratamento da dor crônica no futuro, princi- } \\
\text { palmente com o aumento do número de } \\
\text { estudos. }\end{array}$ \\
\hline Yuan et al, $2016^{43}$ & $\begin{array}{l}\text { Pacientes com osteoartrite refratários ao } \\
\text { tratamento conservador. }\end{array}$ & $\begin{array}{l}\text { O efeito da neurotomia por RF pulsada dos } \\
\text { nervos geniculares é, obviamente, superior } \\
\text { ao uso de injeção de betametasona intra- } \\
\text { articular no tratamento da OA refratária do } \\
\text { joelho, sendo um método eficaz em idosos. }\end{array}$ \\
\hline
\end{tabular}




\section{Discussão}

O presente estudo de revisão avaliou 19 artigos em busca das principais indicações de uso da RF para o tratamento da $\mathrm{OA}$ do joelho.

Relativamente novo no tratamento da dor na OA de joelho, a RF é utilizada em muitos procedimentos que visam o alívio da dor, com resultados eficazes a médio e longo prazo, como demonstrado por estudo realizado por de van Kleef et al., ${ }^{44} \mathrm{o}$ qual avaliou o uso da RF para tratamento da dor lombar crônica.

A OA de Joelho é uma condição clínica que, frequentemente, acarreta incapacidade. Aproximadamente $12 \%$ da população com mais de 60 anos apresentam sintomas relacionados à $\mathrm{OA}$ de joelho. Estudo estima que os custos médicos com a OA variam de US $\$ 1000$ a US $\$ 4100$ por pessoa-ano. ${ }^{45}$

A ATJ ainda é considerada o procedimento cirúrgico padrão-ouro para o tratamento da OA sintomática e refratária ao tratamento conservador no joelho. ${ }^{46}$ Todavia, a ATJ, como procedimento de grande porte, pode gerar complicações, como infecção, instabilidade, lesões neurovasculares, tromboembolismo e, até mesmo, a não melhora da dor. ${ }^{47}$

Na presente revisão, a leitura cuidadosa dos 19 artigos analisados sugere que o uso da RF pode ser considerado como mais uma arma do arsenal terapêutico que pode aliviar a dor crônica do joelho secundária à $\mathrm{OA}$ ou a dor persistente mesmo após realização da ATJ.

Além da melhora da dor, algumas evidências sugerem melhora da função dos pacientes, principalmente em pacientes mais idosos.

Nenhum dos artigos estudados relatam complicações graves relacionadas ao uso da RF. Santana Pineda et al. ${ }^{26}$ concluíram que tal tratamento é seguro e minimamente invasivo, podendo ser realizado em ambiente ambulatorial.

Contudo, existem preocupações quanto à qualidade, ao monitoramento dos resultados e ao tempo no qual o paciente é beneficiado pelo procedimento em questão.

Estudo concluiu que a RF apresenta resultados eficazes, culminando em completo alívio da dor após seis meses de acompanhamento, principalmente quando utilizada a $\mathrm{RF}$ refrigerada, como demonstrado por McCormick et al. ${ }^{31}$

Quando se avaliaram as indicações para o uso da RF, parte dos estudos utilizou a classificação de Kellgren-Lawrence para auxiliá-los.

A classificação de Kellgren-Lawrence figura entre as mais antigas baseadas em radiografias para graduação da OA. É graduada em cinco estágios, sendo o grau zero a ausência de $\mathrm{OA}$ e o grau 4 o mais severo. Como destacado por Rodrigues et al.," ${ }^{48}$ tal classificação é de fácil memorização e interpretação, podendo ser utilizada com segurança. No joelho, utiliza-se apenas radiográfica em incidência anteroposterior, sem a necessidade de ser realizada em apoio monopodálico ou em extensão no joelho. ${ }^{48}$

Autores utilizaram escalas de mensuração de dor e de funcionalidade para ajudar na indicação da neurotomia por $\mathrm{RF}$ dos nervos geniculares. Tais escalas são instrumento de medição geralmente utilizado para quantificar uma característica que não pode ser medida diretamente. Na VAS, a intensidade da dor pode ser avaliada por meio de uma das versões dessa escala que compreende uma linha horizontal de $10 \mathrm{~cm}$, com as extremidades indicando ausência de dor até a pior dor possível. Podem ser determinados valor numérico, descritor verbal e/ou faces de sofrimento, características potencialmente úteis para pacientes que apresentam dificuldades em compreender as escalas numéricas. ${ }^{18}$

Além do estágio da classificação de Kellgren-Lawrence e do uso de escalas de mensuração de dor e de funcionalidade, a refratariedade ao tratamento conservador foi um dos fatores mais citados.

A falha do tratamento conservador no manejo da OA, mesmos naqueles que foram submetidos à ATJ, é, juntamente com o nível de dor do paciente, uma das mais frequentes indicações para realização da neurotomia por RF dos nervos geniculares.

Eyigor et al. ${ }^{42}$ sugerem ainda que o procedimento em questão é eficaz e segura para o tratamento da dor de pacientes com OA de joelho avançada, podendo no futuro ser incluído nas diretrizes de tratamento da dor crônica, principalmente com o aumento do número de estudos na área.

Vale salientar que o uso da RF na propedêutica da gonartrose ainda é um tema recente. As principais evidências sugerem resultados consistentes no primeiro ano de acompanhamento, destacando-se os seis primeiros meses. ${ }^{26,29,31,32}$

Assim, como expresso nas conclusões de Mata et al. ${ }^{37} \mathrm{e}$ Qudsi-Sinclair et al., ${ }^{33}$ é evidente que mais estudos com tamanho amostral maior e acompanhamento mais longo são necessários, principalmente para avaliar a resposta do tratamento a longo prazo.

\section{Considerações Finais}

As principais indicações do uso da RF para o tratamento da $\mathrm{OA}$ do joelho identificadas nos artigos estudados foram: pacientes com osteoartrite grau 3 e 4 da classificação de Kellgren-Lawrence, com dor de moderada a severa e falha do tratamento conservador, principalmente idosos; persistência da dor mesmo após ATJ; pacientes com indicação de ATJ e que se recusam a se submeter ao tratamento cirúrgico. Fica destacado que mais estudos são necessários para corroborarem os achados citados.

Conflitos De Interesse

Os autores declaram não haver conflitos de interesse.

\section{Referências}

1 McAlindon TE, Bannuru RR, Sullivan MC, Arden NK, Berenbaum F, Bierma-Zeinstra SM, et al. OARSI guidelines for the non-surgical management of knee osteoarthritis. Osteoarthritis Cartilage 2014;22(03):363-388

2 Hochberg MC, Altman RD, April KT, Benkhalti M, Guyatt G, McGowan J, et al; American College of Rheumatology. American College of Rheumatology 2012 recommendations for the use of nonpharmacologic and pharmacologic therapies in osteoarthritis of the hand, hip, and knee. Arthritis Care Res (Hoboken) 2012;64 (04):465-474

3 Wannmacher L. Osteoartrose de joelhos Parte II: Evidências sobre abordagens não-medicamentosas. In: Uso racional de medicamentos: temas selecionados. Brasília, DF: Organização Pan-Americana da Saúde/Organização Mundial da Saúde; 2006. (v. 3, n. 4). 
4 Roos EM, Arden NK. Strategies for the prevention of knee osteoarthritis. Nat Rev Rheumatol 2016;12(02):92-101

5 Bruyère $O$, Cooper $C$, Pelletier JP, Branco J, Luisa Brandi $M$, Guillemin F, et al. An algorithm recommendation for the management of knee osteoarthritis in Europe and internationally: a report from a task force of the European Society for Clinical and Economic Aspects of Osteoporosis and Osteoarthritis (ESCEO). Semin Arthritis Rheum 2014;44(03):253-263

6 Herrero-Beaumont G, Roman-Blas JA, Bruyère $\mathrm{O}$, Cooper C, Kanis J, Maggi S, et al. Clinical settings in knee osteoarthritis: Pathophysiology guides treatment. Maturitas 2017;96:54-57

7 Henrotin Y, Raman R, Richette P, Bard H, Jerosch J, Conrozier T, et al. Consensus statement on viscosupplementation with hyaluronic acid for the management of osteoarthritis. Semin Arthritis Rheum 2015;45(02):140-149

8 Herrero-Beaumont G, Ivorra JA, Del Carmen Trabado M, Blanco FJ, Benito P, Martín-Mola E, et al. Glucosamine sulfate in the treatment of knee osteoarthritis symptoms: a randomized, doubleblind, placebo-controlled study using acetaminophen as a side comparator. Arthritis Rheum 2007;56(02):555-567

9 Conaghan P. DMARDs in Osteoarthritis: What is the Evidence? Rheumatology 2014;53(Suppl 1):i12-i13

10 Silveira N, Streck EL. Tratamentos fisioterapêuticos na osteoartrose de joelho: uma revisão. Inova Saúde. 2014;3(01):46-57

11 Moseley JB, O'Malley K, Petersen NJ, Menke TJ, Brody BA, Kuykendall $\mathrm{DH}$, et al. A controlled trial of arthroscopic surgery for osteoarthritis of the knee. N Engl J Med 2002;347(02):81-88

12 Richmond JC. Surgery for osteoarthritis of the knee. Rheum Dis Clin North Am 2008;34(03):815-825

13 Provenzano DA, Lutton EM, Somers DL. The effects of fluid injection on lesion size during bipolar radiofrequency treatment. Reg Anesth Pain Med 2012;37(03):267-276

14 Cosman ER Jr, Dolensky JR, Hoffman RA. Factors that affect radiofrequency heat lesion size. Pain Med 2014;15(12): 2020-2036

15 Provenzano DA, Liebert MA, Somers DL. Increasing the $\mathrm{NaCl}$ concentration of the preinjected solution enhances monopolar radiofrequency lesion size. Reg Anesth Pain Med 2013;38(02):112-123

16 Bhatia A, Peng P, Cohen SP. Radiofrequency Procedures to Relieve Chronic Knee Pain: An Evidence-Based Narrative Review. Reg Anesth Pain Med 2016;41(04):501-510

17 Braun Filho JL, Braun LM. Radiofrequência na dor crônica Radiofrequency in chronic pain Radiofrecuencia en el dolor crónico. Coluna/Columna 2009;8(02):200-205

18 Price DD, McGrath PA, Rafii A, Buckingham B. The validation of visual analogue scales as ratio scale measures for chronic and experimental pain. Pain 1983;17(01):45-56

19 McConnell S, Kolopack P, Davis AM. The Western Ontario and McMaster Universities Osteoarthritis Index (WOMAC): a review of its utility and measurement properties. Arthritis Rheum 2001; 45(05):453-461

20 Steinert T, Eisele F, Längle G, Albani C, Flammer E, Borbé R. [PGI-I (patient's global impression) as an outcome and quality indicator of psychiatric in-patient treatment: results and concordance with doctor's assessments]. Psychiatr Prax 2010;37(07):343-349

21 Silva AL, Demange MK, Gobbi RG, Silva TF, Pécora JR, Croci AT. Translation and Validation of the Knee Society Score - KSS for Brazilian Portuguese. Acta Ortop Bras 2012;20(01):25-30

22 Murray DW, Fitzpatrick R, Rogers K, Pandit H, Beard DJ, Carr AJ, et al. The use of the Oxford hip and knee scores. J Bone Joint Surg Br 2007;89(08):1010-1014

23 Hawker GA, Mian S, Kendzerska T, French M. Measures of adult pain: Visual Analog Scale for Pain (VAS Pain), Numeric Rating Scale for Pain (NRS Pain), McGill Pain Questionnaire (MPQ), Short-Form McGill Pain Questionnaire (SF-MPQ), Chronic Pain Grade Scale (CPGS), Short Form-36 Bodily Pain Scale (SF-36 BPS), and Measure of Intermittent and Constant Osteoarthritis Pain (ICOAP). Arthritis Care Res (Hoboken) 2011;63(Suppl 11):S240-S252
24 Goldberg D, Bridges K, Duncan-Jones P, Grayson D. Detecting anxiety and depression in general medical settings. BMJ 1988;297 (6653):897-899

25 Kirdemir P, Çatav S, Alkaya Solmaz F. The genicular nerve: radiofrequency lesion application for chronic knee pain. Turk J Med Sci 2017;47(01):268-272

26 Santana Pineda MM, Vanlinthout LE, Moreno Martín A, van Zundert J, Rodriguez Huertas F, Novalbos Ruiz JP. Analgesic Effect and Functional Improvement Caused by Radiofrequency Treatment of Genicular Nerves in Patients With Advanced Osteoarthritis of the Knee Until 1 Year Following Treatment. Reg Anesth Pain Med 2017;42(01):62-68

27 Sarı S, Aydın ON, Turan Y, Özlülerden P, Efe U, Kurt Ömürlü İ. Which one is more effective for the clinical treatment of chronic pain in knee osteoarthritis: radiofrequency neurotomy of the genicular nerves or intra-articular injection? Int J Rheum Dis 2018;21(10):1772-1778. Doi: 10.1111/1756-185X.12925

28 Kesikburun S, Yaşar E, Uran A, Adigüzel E, Yilmaz B. UltrasoundGuided Genicular Nerve Pulsed Radiofrequency Treatment For Painful Knee Osteoarthritis: A Preliminary Report. Pain Physician 2016;19(05):E751-E759

29 Bellini M, Barbieri M. Cooled radiofrequency system relieves chronic knee osteoarthritis pain: the first case-series. Anaesthesiol Intensive Ther 2015;47(01):30-33

30 Davis T, Loudermilk E, DePalma M, Hunter C, Lindley D, Patel N, et al. Prospective, Multicenter, Randomized, Crossover Clinical Trial Comparing the Safety and Effectiveness of Cooled Radiofrequency Ablation with Corticosteroid Injection in the Management of Knee Pain From Osteoarthritis. Reg Anesth Pain Med 2018;43(01):84-91

31 McCormick ZL, Korn M, Reddy R, Marcolina A, Dayanim D, Mattie $\mathrm{R}$, et al. Cooled Radiofrequency Ablation of the Genicular Nerves for Chronic Pain due to Knee Osteoarthritis: Six-Month Outcomes. Pain Med 2017;18(09):1631-1641

32 Iannaccone F, Dixon S, Kaufman A. A Review of Long-Term Pain Relief after Genicular Nerve Radiofrequency Ablation in Chronic Knee Osteoarthritis. Pain Physician 2017;20(03):E437-E444

33 Qudsi-Sinclair S, Borrás-Rubio E, Abellan-Guillén JF, Padilla Del Rey ML, Ruiz-Merino G. A Comparison of Genicular Nerve Treatment Using Either Radiofrequency or Analgesic Block with Corticosteroid for Pain after a Total Knee Arthroplasty: A Double-Blind, Randomized Clinical Study. Pain Pract 2017;17(05):578-588

34 Sarı S, Aydın ON, Turan Y, Şen S, Özlülerden P, Ömürlü İK, et al. Which imaging method should be used for genicular nerve radio frequency thermocoagulation in chronic knee osteoarthritis? J Clin Monit Comput 2017;31(04):797-803

35 Shen WS, Xu XQ, Zhai NN, Zhou ZS, Shao J, Yu YH. Radiofrequency Thermocoagulation in Relieving Refractory Pain of Knee Osteoarthritis. Am J Ther 2017;24(06):e693-e700

36 Mogahed M, Mohamed R, Mohamed Refaat H. Intraarticular Pulsed Radiofrequency vs. Radiofrequency Neurotomy in Patients with Chronic Knee Pain due to Osteoarthritis (OA). J Anesth Clin Res 2017;8(10):2-6

37 Mata J, Valentí P, Hernández B, Mir B, Aguilar JL. Study protocol for a randomised controlled trial of ultrasound-guided pulsed radiofrequency of the genicular nerves in the treatment of patients with osteoarthritis knee pain. BMJ Open 2017;7(11): e016377

38 Gulec E, Ozbek H, Pektas S, Isik G. Bipolar Versus Unipolar Intraarticular Pulsed Radiofrequency Thermocoagulation in Chronic Knee Pain Treatment: A Prospective Randomized Trial. Pain Physician 2017;20(03):197-206

39 Masala S, Fiori R, Raguso M, Morini M, Calabria E, Simonetti G. Pulse-dose radiofrequency for knee osteoartrithis. Cardiovasc Intervent Radiol 2014;37(02):482-487

40 Hashemi M, Nabi BN, Saberi A, Sedighinejad A, Haghighi M, Farzi F, et al. The Comparison between two methods for the relief of knee osteoarthritis pain: radiofrequency and intra-periarticular ozone 
injection: a clinical trial study. Int J Med Res Health Sci. 2016;5 (7S):539-546

41 Ramírez Ogalla I, Martín AM, Santana Pineda MM, Rodríguez Huertas F. Eficacia de la radiofrecuencia convencional de geniculados para el tratamiento del dolor en gonartrosis moderadasevera. Rev Soc Esp Dolor. 2014;21(04):212-218

42 Eyigor C, Eyigor S, Akdeniz S, Uyar M. Effects of intra-articular application of pulsed radiofrequency on pain, functioning and quality of life in patients with advanced knee osteoarthritis. J Back Musculoskeletal Rehabil 2015;28(01):129-134

43 Yuan Y, Shen W, Han Q, Liang D, Chen L, Yin Q, et al. Clinical observation of pulsed radiofrequency in treatment of knee osteoarthritis. Int J Clin Exp Med 2016;9(10):20050-20055

44 van Kleef M, Barendse GA, Kessels A, Voets HM, Weber WE, de Lange S. Randomized trial of radiofrequency lumbar facet denervation for chronic low back pain. Spine 1999;24(18): 1937-1942

45 Losina E, Walensky RP, Kessler CL, Emrani PS, Reichmann WM, Wright EA, et al. Cost-effectiveness of total knee arthroplasty in the United States: patient risk and hospital volume. Arch Intern Med 2009;169(12):1113-1121, discussion $1121-1122$

46 Mancuso CA, Ranawat CS, Esdaile JM, Johanson NA, Charlson ME. Indications for total hip and total knee arthroplasties. Results of orthopaedic surveys. J Arthroplasty 1996;11(01):34-46

47 Cheung A. Complications of total knee arthroplasty. Curr Orthop 2008;22(04):274-283

48 Rodrigues AA, Karam FC, Scorsatto C, Martins C, Pires LA. Análise da reprodutibilidade da classificação de Kellgren e Lawrence para osteoartrose do joelho. Rev AMRIGS. 2012;56(02):107-110 